Published in final edited form as:

Neurotoxicology. 2007 May ; 28(3): 569-575.

\title{
Kinetic Tremor:
}

\section{Differences Between Smokers and Non-smokers}

Elan D. Louis, MD, MSc

Gertrude H. Sergievsky Center, and Department of Neurology, College of Physicians and Surgeons, Columbia University, New York, NY, USA.

\begin{abstract}
Tremor is among the acute effects of nicotine exposure. Published studies have focused on smokingrelated postural (static) hand tremor rather than kinetic tremor (tremor during hand use), and gender differences in smoking-related tremor have not been examined. In a group of adults who were sampled from a population (mean $\pm \mathrm{SD}=65.7 \pm 11.5$ years, range $=18$ - 92 years), the investigator assessed whether the severity of postural and kinetic tremors differed in smokers versus non-smokers, and whether this difference was influenced by gender. Twenty-seven $(9.9 \%)$ of 273 subjects were current smokers. Greater tremor was observed in smokers than non-smokers during a variety of activities (drawing a spiral, using a spoon, finger-nose-finger maneuver, all $p<0.05$ ) and smokers had a higher total tremor score than non-smokers ( $5.15 \pm 3.06$ vs. $3.41 \pm 2.88, \mathrm{p}<0.01)$, even after adjusting for age, caffeine intake and other potential confounding factors. The difference between smokers and non-smokers in terms of hand tremor was more apparent in women than in men. In women, the number of cigarettes smoked on the day of testing was weakly correlated with the total tremor score $(r=0.17, p=0.03)$. In summary, smokers had more kinetic hand tremor than nonsmokers. This difference between smokers and non-smokers was more apparent in women than in men. These results suggest that smoking habits should be considered carefully in order to avoid overor underestimating the effects of occupational and non-occupational exposures to other tremorproducing neurotoxins.
\end{abstract}

\section{Keywords}

tremor; smoking; nicotine; gender

\section{Introduction}

It is well-known that tremor is a common manifestation of exposure to several neurotoxins, including lead, pesticides, and harmane, and that this tremor can be disabling (Seshia et al. 1978;Valpey et al. 1978; Louis et al. 2002;Louis et al. 2006). The tremor may take on several forms, including tremors that occur while the arms are at rest and kinetic tremors, which occur while the arms are performing purposeful movements such as eating or writing. Kinetic tremors are of particular concern because they may interfere with the performance of daily activities.

Smoking can have numerous physiological effects. Among these is tremor, which could be the result of nicotine exposure (Maykoski et al. 1976;Lippold et al. 1978; Lippold et al.

Correspondence: Dr. Elan Louis, Unit 198, Neurological Institute, 710 West 168th Street, New York, NY, 10032, USA. Tel: (212) 305 - 9194, FAX: (212) 305 - 1304, Email: EDL2@ columbia.edu.

Publisher's Disclaimer: This is a PDF file of an unedited manuscript that has been accepted for publication. As a service to our customers we are providing this early version of the manuscript. The manuscript will undergo copyediting, typesetting, and review of the resulting proof before it is published in its final citable form. Please note that during the production process errors may be discovered which could affect the content, and all legal disclaimers that apply to the journal pertain. 
1980; Shiffman et al. 1983; Gomita et al. 1991). When assessing populations for the tremorproducing effects of neurotoxic compounds like lead and pesticides, smoking habits should also be considered (Ellingsen et al. 2006).

Human studies of the effects of nicotine have been limited to the assessment of postural tremor, usually examining static tremor in a single finger of an outstretched hand. These studies have not assessed kinetic hand tremor, which is tremor occurring during common daily hand activities such as writing and pouring. Furthermore, these previous studies have relied on tremor recordings rather than assessing clinically detectable tremor using a simple clinical rating scale. Finally, gender differences in tremor have not been examined despite evidence that the physiological effects of cigarette smoke might differ in men and women (Dluzen and Anderson 1997).

A detailed clinical assessment of tremor was performed in 273 men and women without essential tremor. Data on smoking habits were also collected. This opportunity was used to: (1) compare smokers to non-smokers in terms of the severity of both static tremor as well as kinetic tremor in different conditions, (2) assess whether any differences between smokers and non-smokers were influenced by gender.

\section{Methods}

\section{Study Design and Study Subjects}

An ongoing case-control study of the environmental epidemiology of essential tremor is being conducted at Columbia University Medical Center (2000 - present). The goal of this study is to examine whether there is an association between a variety of putative neurotoxins, including lead, beta-carboline alkaloids, and pesticides, and essential tremor (Louis et al. 2002;Louis et al. 2006). Essential tremor cases are recruited from the outpatient department of the Medical Center's Neurological Institute. Control subjects are identified from the population using random digit telephone dialing and are ascertained from the same source population in the New York Tri-state region (i.e. the same set of zip codes in New York, New Jersey and Connecticut) as are the cases; none has essential tremor, Parkinson's disease or dystonia. These control subjects are frequency matched to cases on five year age strata, gender and ethnicity. Among 449 potential control subjects, $273(60.8 \%)$ agreed to participate and were enrolled. The Columbia University Medical Center Internal Review Board approved of all study procedures and written informed consent was obtained at the time of enrollment. The present analyses were focused on the control subjects. The goal of these analyses was to assess whether smokers had more hand tremor than non-smokers. The study was not designed to capture the acute effects of smoking. In other words, tremor was not measured in smokers before and immediately after they smoked a cigarette, although some data are provided on the association between the number of cigarettes smoked on the day of testing and the severity of tremor.

\section{History and Examinations}

Once enrolled, control subjects were evaluated in person by a trained tester either in their homes or in an outpatient setting at the Medical Center. On the day of testing they were not asked to refrain from eating, drinking or other habits like smoking. The tester administered clinical questionnaires and performed a videotaped tremor examination (Louis et al. 2002; Louis et al. 2006). Using the clinical questionnaires, the trained tester collected data on age, gender, race (coded as white vs. non-white), number of rooms in their home (a socioeconomic status indicator), occupational category, and smoking history, including current use of cigarettes, years smoked, pack-years, and number of cigarettes smoked on the day of testing. Current cigarette smokers were defined as those who answered affirmatively to the question, "Do you currently smoke cigarettes?" Data on current medications were collected. These included the 
following tremor inducing-medications: tricyclic antidepressants, selective serotonin reuptake inhibitors, digoxin, oral hypoglycemics, nitrates, steroids, asthma inhalers, lithium, valproic acid, diet pills, birth control pills, theophylline, thyroxine, and estrogen replacement therapy. Data on current diet were collected using a semiquantitative food-frequency questionnaire (Willett et al. 1985), including questions on coffee, tea, and soda consumption, which were converted into $\mathrm{mg}$ of caffeine per day. These data were important because caffeine ingestion can increase the severity of low-amplitude finger tremors (Wharrad et al. 1985). Subjects were also asked to note whether they had used an asthma inhaler on the day of testing. The severity of illness in 14 body systems was rated using the Cumulative Illness Rating Scale; each system received a rating from 0 to 3 , with 3 indicating severe illness. The range of scores was from 0 to 42 (Linn et al. 1968).

For the main measure of tremor, each subject had a videotaped tremor examination. This included sustained arm sustention, which was a test of postural tremor. It also included the following five tests to elicit kinetic tremor: pouring water between two cups, drinking water from a cup, using a spoon to drink water, finger-nose-finger maneuver, and drawing a spiral on a sheet of paper (Louis et al. 2002; Louis et al. 2006). Each of the six tests was performed with the dominant arm and then the nondominant arm, resulting in 12 tests total (Louis et al. 2002). Each videotape was reviewed by a neurologist specializing in movement disorders (E.D.L.) who was blinded to clinical information on smoking status, diet and medications. The tremor was rated during each test using a clinical rating scale. The ratings were as follows: 0 $=$ no tremor, $1=$ low amplitude tremor was barely perceivable or was intermittent, $2=$ tremor was of moderate amplitude and was usually present and was clearly oscillatory, $3=$ large amplitude, jerky tremor resulting in difficulty completing the task due to spilling or inability to hold a pen to paper. Thereby, each participant was assigned a tremor score ranging from 0 to 18 for the dominant hand and 0 to 18 for the non-dominant hand. The total tremor score, ranging from 0 to 36, was the sum of these two (Louis et al. 2002). The reliability of this videotape-based rating has been demonstrated by weighted kappa statistics of $0.62-0.78$, indicating substantial inter-rater agreement (Louis et al. 1998) and the score correlates with other objective measures of tremor severity (Louis et al. 1999).

The Klove-Matthews Motor Steadiness Battery was administered as a secondary measure of tremor severity. This was the secondary rather than primary measure of tremor because a variety of motor problems such as myoclonus, chorea, and weakness may lead to poor performance. This contrasts with the primary measure during which only tremor, if visualized, was rated. This test consisted of a two-part, 15-minute test protocol. In the first part, a Groove Type Steadiness Tester (Model 32010, Lafayette Instrument, Lafayette, IN) was used to assess kinetic tremor. The unit consisted of two adjustable steel plates which formed the sides of a progressively narrowing groove. The distance between the steel plates was 0.625 inches at one end and 0.125 inches at the other end. The unit was placed flatly on the table and oriented so that the 0.625 inch opening was on the subject's left. The subject moved a hand-held metaltipped stylus (diameter $=0.0625$ inches, Model 32100, Lafayette Instrument, Lafayette, IN) horizontally from left to right through the gradually narrowing groove without touching the steel sides of the groove. Any contact between the stylus and the steel wall of the groove completed a circuit and was recorded by a battery operated Silent Impulse Counter (Model 58023, Lafayette Instrument, Lafayette IN). The Impulse Counter recorded the number of contacts between the stylus and the wall. In the second part, a Nine Hole Steadiness Tester (Model 32011, Lafayette Instrument, Lafayette, IN) was used to assess postural tremor. This consisted of a vertical metal plate with holes of gradually diminishing size. The subject was asked to hold the hand-held metal tipped stylus in each of three holes (diameters $=0.312,0.25$, 0.187 inches) for 10 seconds without touching the metal sides. Any contact between the metaltipped stylus and the steel wall of the hole completed a circuit and was recorded by a battery operated Silent Impulse Counter. The Impulse Counter recorded the number of contacts 
between the stylus and the wall. The testing procedure has been described in detail (Louis et al. 2000).

\section{Additional Assessments of Exposure to Neurotoxins}

Because control subjects were enrolled in a study of the environmental epidemiology of essential tremor, exposures to a variety of tremor-producing toxins were assessed. These tremor-producing toxins were lead, harmane, and six organochlorine pesticides (p,p'-DDE, $\mathrm{p}, \mathrm{p}$ '-DDT, $\beta$-hexachlorocyclohexane, oxychlordane, trans-nonachlor, and dieldrin). As documented previously, blood lead concentration was analyzed using graphite furnace atomic absorption spectrophotometry (Fernandez and Hilligoss 1982;Louis et al. 2003). The high performance liquid chromatography method to quantify blood harmane concentration was described previously (Louis et al., 2002;Zheng et al., 2000). Serum pesticide concentrations were determined by gas chromatography with electron capture detection following an organochlorine-specific cleanup of the lipid fraction of serum, as detailed elsewhere (Wolff et al. 1991; Wolff et al. 1982; Wolff et al. 2005; Louis et al. 2006).

\section{Power Analysis}

A post hoc power analysis indicated that the sample size, 246 non-smokers and 27 smokers, provided $82.9 \%$ power to detect a total tremor score that was $\geq 50 \%$ higher among smokers than non-smokers. These calculations assumed alpha $=0.05$ and the mean \pm SD total tremor score among non-smokers $=3.41 \pm 2.88$. A similar analysis for subjects $<60$ years of age $(7$ smokers and 55 non-smokers) indicated that there was limited power (30.1\%) to detect a $>$ $50 \%$ difference in total tremor score between younger smokers and non-smokers; in this younger age-group, there was adequate power ( $82.7 \%)$, however, to detect a doubling of total tremor scores in smokers vs. non-smokers.

\section{Statistical Analyses}

All analyses were performed in SPSS, Version 13.0. The total tremor score and Klove Matthews Test scores were not normally distributed. Therefore, a non-parametric test, the Mann Whitney U test, was used to compare these scores in smokers vs. non-smokers. A Spearman's correlation coefficient, $r$, was used to assess the associations between total tremor score and years smoked, pack-years, and number of cigarettes smoked on the day of testing. For a linear regression analysis, the total tremor score was $\log$ transformed $\left(\log _{10}\right)$. Because the total tremor score had a value of 0 in thirty-eight of 273 subjects, 0.5 was added to the value in all 273 subjects prior to this $\log$ transformation. In linear regression analyses, $\log _{10}$ total tremor score was the dependent variable and current smoker (yes vs. no) was the independent variable, adjusting for a variety of confounding variables in different models.

\section{Results}

There were 273 subjects; none had essential tremor, Parkinson's disease, or dystonia by history or as determined by an assessment of the videotape examination. Twenty-seven $(9.9 \%)$ were current smokers and $246(90.1 \%)$ were not. The mean \pm SD age was $65.7 \pm 11.5$ years, with the range being 18 - 92 years. Nineteen $(7.0 \%)$ subjects were $<40$ years of age and $33(12.1 \%)$ were $<50$ years of age. Smokers and non-smokers were similar in terms of age, gender, and several other characteristics (Table 1). Smokers had fewer years of education, fewer rooms in their homes, and consumed more caffeine than non-smokers (Table 1). Smokers and nonsmokers did not differ with respect to the serum concentration of any of the six organochlorine pesticides or the blood harmane concentration; however, blood lead concentration was higher in smokers than non-smokers ( $3.7 \pm 1.7$ vs. $2.6 \pm 1.6, \mathrm{p}=0.003)$. 
The total tremor score as well as the dominant and non-dominant hand tremor scores were greater in smokers vs. non-smokers (Table 2, Figure). Tremor during several hand activities (using a spoon, finger-nose-finger maneuver, and drawing a spiral) was more pronounced in smokers than in non-smokers (e.g., the mean tremor score while drawing a spiral was 2.17 times higher in smokers than non-smokers, Table 2).

Seven (25.9\%) smokers vs. 29 (11.8\%) non-smokers had hand tremor rated as mild or greater while drawing a spiral with their dominant hand $(\mathrm{p}=0.04)$. Similarly, a larger proportion of smokers than non-smokers had dominant hand tremor rated as mild or greater while using a spoon: $15(55.6 \%)$ vs. $75(30.7 \%, \mathrm{p}=0.009)$ and while performing the finger-nose-finger maneuver: $18(66.7 \%)$ vs. $112(46.5 \%, \mathrm{p}=0.05)$.

The mean total tremor score was higher in men than women: $4.49 \pm 3.34$ vs. $3.04 \pm 2.53$ (p < 0.001). The sample was stratified by gender and differences between smokers and non-smokers were examined within each stratum separately (Table 3 ). The difference between smokers and non-smokers was more apparent in the female than the male stratum (Table 3, Figure); the gender difference between smokers and non-smokers was not due to the confounding effects of blood lead concentration, which was similar in men and women. Similarly, the gender difference was not due to the effects of estrogen replacement therapy; two of the seventeen women who were smokers were on estrogen replacement therapy and their total tremor score was similar to that of the 15 female smokers not on this therapy $(4.00 \pm 1.41$ vs. $5.13 \pm 3.42$, $\mathrm{p}=0.82$ ). The sample contained a small number of younger subjects, including 2 smokers and 31 non-smokers < 50 years of age. While total tremor score trended to be higher in the two smokers $(5.0 \pm 2.8$ vs. $3.5 \pm 3.3)$, the small numbers precluded a meaningful statistical comparison. There were 7 smokers and 55 non-smokers $<60$ years of age. Here too, there was a trend for the total tremor score to be higher among smokers $(4.7 \pm 2.2$ vs. $3.7 \pm 3.1)$, although the small sample limited statistical power.

Linear regression analyses were performed to assess the possible impact of confounding variables on the observed association between smoking and tremor score. In these analysis, $\log _{10}$ total tremor score was the dependent variable and current smoker (yes vs. no) was the independent variable. In the initial analyses, which did not adjust for the effects of other confounders, smoking was associated with $\log _{10}$ total tremor score (beta $=0.25, \mathrm{p}=0.002$, Table 4). In an analysis that adjusted for ten potential confounding variables (age, gender, caffeine consumption per day in $\mathrm{mg}$, taking a tremor-inducing medication, used an asthma inhaler on the day of testing, Cumulative Illness Rating Scale score, education, number of rooms in home, white race, occupational category), the association between smoking and $\log _{10}$ total tremor score remained robust (beta $=0.21, \mathrm{p}=0.01$, Table 4 ). After also including the number of cigarettes smoked on the day of testing in the adjusted model, the association between smoking and $\log _{10}$ total tremor score remained robust (beta $=0.28, p=0.03$ ) whereas there was no association between the number of cigarettes smoked on the day of testing and $\log _{10}$ total tremor score (beta $=-0.02, p=0.51$ ). In separate linear regression analyses that adjusted for blood or serum concentrations of each of the other neurotoxins, including lead, the association between smoking and $\log _{10}$ total tremor score remained robust (i.e., all $\mathrm{p}$ values $<0.05$, Table 4).

Among the 27 smokers, the mean \pm SD number of years smoked was $46.3 \pm 13.3$ years and the mean \pm SD number of pack-years was $43.1 \pm 24.3$. Among smokers, neither the number of years smoked $(\mathrm{r}=0.03, \mathrm{p}=0.91)$ nor pack-years was correlated with the total tremor score $(\mathrm{r}=0.39, \mathrm{p}=0.30)$.

Twenty-two $(81.5 \%)$ of 27 smokers had smoked on the day of testing, with the mean \pm SD number of cigarettes smoked being $3.7 \pm 3.0$. The number of cigarettes smoked on the day of 
testing was weakly correlated with the total tremor score in women $(\mathrm{r}=0.17, \mathrm{p}=0.03)$ but not in men $(r=0.09, p=0.37)$.

\section{Discussion}

A number of toxins like lead, harmane, and pesticides are associated with tremor; the severity of these action tremors ranges from mild (low amplitude) to marked (Louis et al. 2002; Louis et al.. 2003). Several studies have demonstrated higher blood concentrations of several toxins in subjects with tremor (Louis et al. 2002;Louis et al.. 2003). Hence, assessing the effects of these toxins on the nervous system is important. These results show that smokers had higher tremor scores than non-smokers and that this difference between smokers and non-smokers was more apparent in women than in men. These data suggest that smoking habits should be taken into account when estimating the effects of occupational or non-occupational exposures to other tremor producing toxins. Failure to do so could result in over- or underestimates of the tremor-producing effects of these other toxins. In other words, observed differences in tremor severity, or lack of observed differences in tremor severity between toxin exposed vs. unexposed groups could be due to the confounding effects of smoking-related tremor.

Smoking-related tremor is thought to be mediated by nicotine-induced catecholamine release. Indeed, nicotine tremor may be blocked in laboratory animals with the use of adrenergic blocking agents (Cahen et al. 1953; Shiffman et al. 1983; Quattrocki et al. 2000). Although it is surmised that observed differences between smokers and non-smokers are due to the effects of nicotine, this is an extrapolation since nicotine was never measured in these study subjects.

In the current study, smokers had more tremor than non-smokers during a number of clinicallyapparent and common hand activities such as using a spoon and writing. More than twice as many (25.9\% vs. $11.8 \%)$ smokers vs. non-smokers had hand tremor rated as mild or greater while writing with their dominant hand. The difference between smokers and non-smokers in terms of hand tremor was more apparent in women than in men. The explanation for this observed gender difference is not clear, however, previous investigators have shown that there are marked differences in the effects of nicotine on males and females (Dluzen and Anderson 1997). In one study, nicotine-evoked catecholamine release was increased in estrogen treated females (Dluzen and Anderson 1997). Given the tremor-produced effect of catecholamines (Young et al. 1975), these data would support the current results.

In this study, the primary interest was the non-acute effects of smoking on the magnitude of tremor. The study was not designed to capture the acute effects of smoking; tremor was not measured in smokers before and immediately after they smoked a cigarette. Moreover, the number of cigarettes smoked on the day of testing did not account for the difference in total tremor score between smokers and non-smokers, suggesting that the observed difference was not due to the acute (i.e., immediate) effects of smoking. Also, the emphasis was not on the chronic/cumulative effects of smoking. It was not hypothesized that chronic smoking would result in cumulative increases in tremor severity over time and therefore, there was no expectation that smoking duration would be associated with tremor severity. Indeed, the number of years smoked and the number of pack years were not correlated with the total tremor score. The emphasis was to compare smokers and non-smokers in terms of the severity of their tremor; tremor was assessed at one point in time and this time point was not based upon the timing of their last cigarette.

Overall, women had lower mean tremor scores than men. Previous investigators have demonstrated that physiological tremor has a higher frequency in women than men and that this is likely due to smaller hand volume in women (Raethjen et al. 2000). Because tremor frequency and amplitude are often inversely related to one another, this suggests that larger 
hand volumes in men could result in higher tremor amplitudes. Unfortunately, data were not collected on hand volume in the current set of control subjects so that it was not possible to directly assess these relationships in the current dataset.

This study had limitations. Smoking was assessed by self-report and there was no measurement of urine cotinine concentration as an independent measure of smoking habits. In addition, some analyses were stratified. As is often the case with stratified analyses, the power to detect differences between groups diminishes. Despite this potential limitation, several differences were apparent in the female stratum when comparing smokers to non-smokers. These differences included the total tremor score, tremor score in each hand, tremor while using a spoon, tremor while performing the finger-nose-finger maneuver, tremor while drawing a spiral, and Nine Hole Steadiness Tester results. Although there was a trend for total tremor score to be higher in younger smokers than younger non-smokers, the small number of younger subjects only provided adequate power to detect a doubling of total tremor score in younger smokers. Hence, the ability to generalize these findings to younger persons is limited. Finally, although smoker and non-smokers were similar in many respects, including age, gender and medication use, smokers had fewer years of education, fewer rooms in their homes, and consumed more caffeine than did non-smokers. An attempt was made to adjust for these difference in the regression analyses.

This study had a number of strengths. First, studies to date have assessed the effects of nicotine on static tremor; in the current study kinetic tremor was assessed in smokers and non-smokers during a variety of common daily hand activities such as writing, pouring etc. Second, previous studies have limited their approach to quantitative computerized tremor analysis. While this approach adds precision to the measurement of tremor amplitude, it does not ensure that the results are clinically meaningful. In other words, while some tremors are detectable using quantitative computerized tremor analysis, they may not be visible to a clinician who is examining the patient. Similarly, these tremors may not be of functional significance in the sense that the patient may not notice them or complain about them. In the current report, the focus was on tremor that was clinically detectable using a simple clinical rating scale. Furthermore, each videotaped tremor examination was reviewed and tremor was rated by a neurologist specializing in movement disorders. Finally, the influences of gender on hand tremor in smokers and non-smokers has not previously been examined.

In summary, smokers had more hand tremor than non-smokers; this tremor included kinetic tremors during a variety of tasks. These results highlight the importance of considering smoking habits when assessing the tremor-producing effects of various neurotoxins.

\section{Acknowledgments}

R01 NS39422, P30 ES09089, P42 ES 10349, and RR00645 (General Clinical Research Center)(NIH, Bethesda, MD). The author gratefully acknowledges the work of Joseph Graziano Ph.D. in performing the blood lead analyses, Wei Zheng Ph.D., in performing the blood harmane analyses, and Mary Wolff $\mathrm{PhD}$, in performing the serum pesticides analyses.

\section{References}

Cahen RL, Thomas JM, Irede KM. Nicotinolytic drugs. II. Action of adrenergic blocking agents on nicotine-induced tremors. J Pharmacol Exp Ther 1953;107:424-436. [PubMed: 13053406]

Dluzen DE, Anderson LI. Estrogen differentially modulates nicotine-evoked dopamine release from the striatum of male and female rats. Neurosci Lett 1997;230:140-142. [PubMed: 9259484]

Ellingsen DG, Bast-Pettersen R, Efskind J, et al. Hand tremor related to smoking habits and the consumption of caffeine in male industrial workers. Neurotoxicology 2006;27:525-533. [PubMed: 16574229] 
Fernandez F, Hilligoss D. An improved graphite furnace method for the determination of lead in blood using matrix modification and the L'vov platform. Atomic Spectr 1982;3:130-131.

Gomita Y, Suemaru K, Furono K, Araki Y. Tail-tremor induced by exposure to cigarette smoke in rats. Pharmacol Biochem Behav 1991;40:453-455. [PubMed: 1805247]

Linn BS, Linn MW, Gurel L. Cumulative illness rating scale. J Am Geriatr Soc 1968;16:622-626. [PubMed: 5646906]

Lippold OC, Williams EJ, Wilson CG. Finger tremor and cigarette smoking. Br J Clin Pharmacol 1980;10:83-86. [PubMed: 7397058]

Lippold OC, Wilson CG. The effect of cigarette smoking on finger tremor. Br J Clin Pharmacol 1978;6:P457-458.

Louis ED, Ford B, Bismuth B. Reliability between two observers using a protocol for diagnosing essential tremor. Mov Disord 1998;13:287-293. [PubMed: 9539343]

Louis ED, Wendt KJ, Albert SM, Pullman SL, Yu Q, Andrews H. Validity of a performance-based test of function in essential tremor. Arch Neurol 1999;56:841-846. [PubMed: 10404986]

Louis ED, Yousefzadeh E, Barnes LF, Yu Q, Pullman SL, Wendt KJ. Validation of a portable instrument for assessing tremor in epidemiological field studies. Mov Disord 2000;15:95-102. [PubMed: 10634247]

Louis ED, Zheng W, Jurewicz EC, Watner D, Chen J, Factor-Litvak P, Parides M. Elevation of blood $\beta$-carboline alkaloids in essential tremor. Neurology 2002;59:1940-1944. [PubMed: 12499487]

Louis ED, Jurewicz EC, Applegate L, et al. Association between essential tremor and blood lead concentration. Environ Health Perspect 2003;111:1707-1711. [PubMed: 14594619]

Louis ED, Factor-Litvak P, Parides M, Andrews L, Santella RM, Wolff MS. Organochlorine pesticide exposure in essential tremor: A case-control study using biological and occupational exposure assessments. Neurotoxicol 2006;27:579-586.

Lynch WJ, Roth ME, Carroll ME. Biological basis of sex differences in drug abuse: preclinical and clinical studies. Psychoparmacol 2002;164:121-137.

Maykoski KA, Rubin MB, Day AC. Effect of cigarette smoking on postural muscle tremor. Nurs Res 1976;25:39-43. [PubMed: 1044041]

Quattrocki E, Baird A, Yurgelun-Todd D. Biological aspects of the link between smoking and depression. Harvard Rev Psychiatry 2000;8:99-110.

Raethjen J, Pawlas F, Lindemann M, Wenzelburger R, Deuschl G. Determinants of physiologic tremor in a large normal population. Clin Neurophysiol 2000;11:1825-1837. [PubMed: 11018499]

Seshia SS, Rjani KR, Boeckx RL, Chow PN. The neurological manifestations of chronic inhalation of leaded gasoline. Dev Med Child Neurol 1978;20:323-334. [PubMed: 97111]

Shiffman SM, Gritz ER, Maltese J, Lee MA, Schneider NG, Jarvik ME. Effects of cigarette smoking and oral nicotine on hand tremor. Clin Pharmacol Ther 1983;33:800-805. [PubMed: 6851411]

Valpey R, Sumi SM, Copass MK, Goble GJ. Acute and chronic progressive encephalopathy due to gasoline sniffing. Neurology 1978;28:507-510. [PubMed: 565491]

Wharrad HJ, Birmingham AT, Madonald IA, Inch PJ, Mead JL. The influence of fasting and of caffeine intake on finger tremor. Eur J Clin Pharmacol 1985;29:37-43. [PubMed: 4054205]

Willett WC, Sampson L, Stampfer MJ, et al. Reproducibility and validity of a semiquantitative food frequency questionnaire. Am J Epidemiol 1985;122:51-65. [PubMed: 4014201]

Wolff MS, Fischbein A, Thornton J, Rice C, Lilis R, Selikoff IJ. Body burden of polychlorinated biphenyls among persons employed in capacitor manufacturing. Int Arch Occup Environ Hlth 1982;49:199-208.

Wolff MS, Rivera M, Baker DB. Detection limits of organochlorine pesticides and related compounds in blood serum. Bull Environ Contam Toxicol 1991;47:499-503. [PubMed: 1786432]

Wolff MS, Deych E, Ojo F, Berkowitz GS. Predictors of organochlorines in New York City pregnant women, 1998-2001. Environ Res 2005;97:170-177. [PubMed: 15533333]

Young RR, Growdon JH, Shahani BT. Beta-adrenergic mechanisms in action tremor. N Engl J Med 1975;293:950-953. [PubMed: 1178003] 
Zheng W, Wang S, Guan Y, Louis E. Determination of harmane and harmine in human blood using reversed-phased high-performance liquid chromatography and fluorescence detection. Anal Biochem 2000;279:125-129. [PubMed: 10706780] 


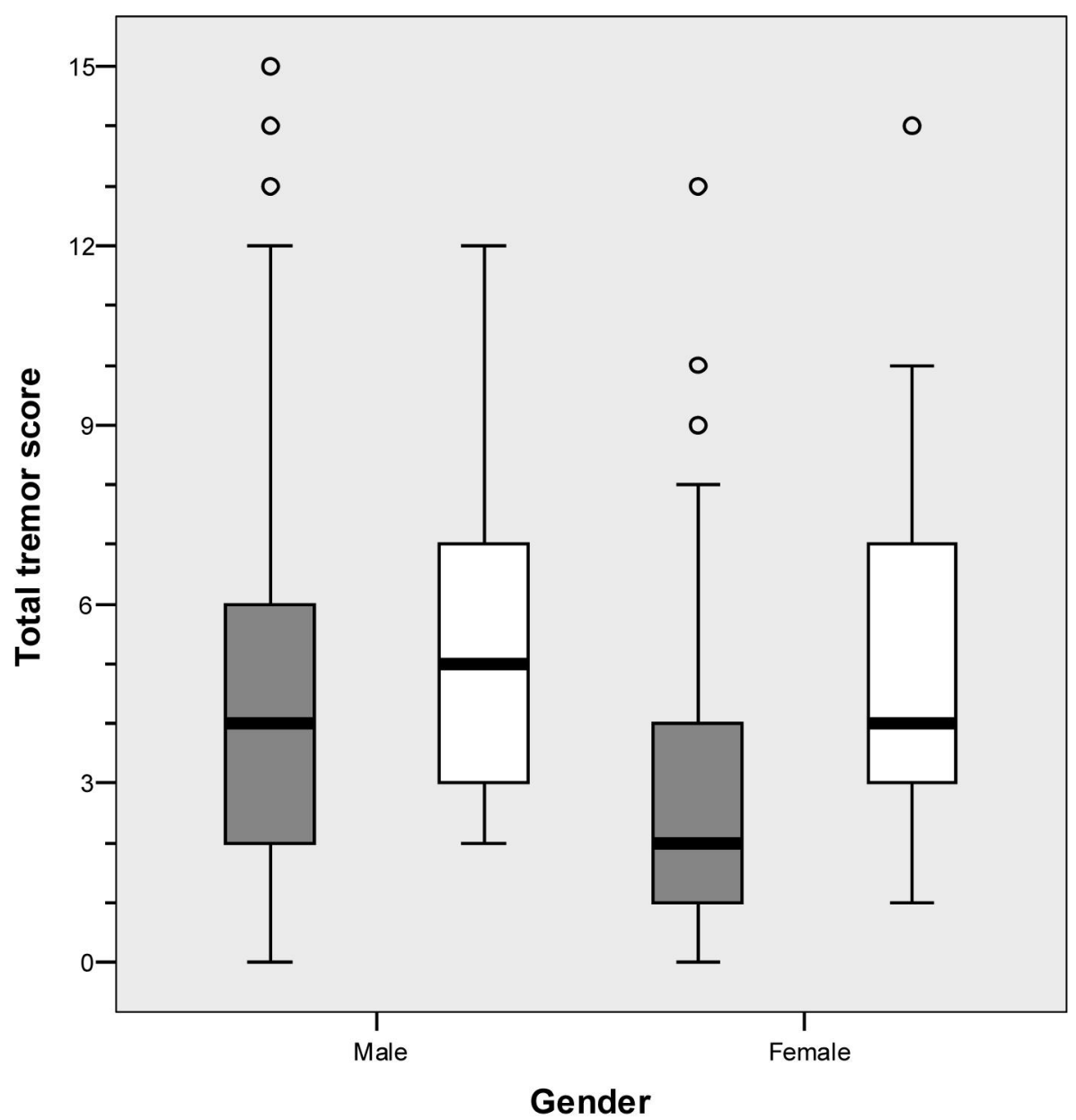

Figure.

Total tremor score by gender. Data on smokers are depicted by the two open bars and data on non-smokers by two shaded bars. The thin vertical line represents the quartile range and the thick vertical bar, the inter-quartile range. The horizontal line represents the median value. 
Table 1

Characteristics of smokers vs. non-smokers

\begin{tabular}{|l|l|l|}
\hline & Current Smokers (N= 27) & Current Non-smokers (N = 246) \\
\hline Age in years & $64.9 \pm 11.5$ & $65.7 \pm 13.8$ \\
\hline Female gender & $17(63.0 \%)$ & $151(61.6 \%)$ \\
\hline Caffeine consumption per day in mg & $347.1 \pm 227.8 * * *$ & $216.4 \pm 202.7$ \\
\hline Taking a tremor-inducing medication & $6(22.2 \%)$ & $50(20.3 \%)$ \\
\hline Used an asthma inhaler on the day of testing & $6(7.4 \%)$ & $6(2.5 \%)$ \\
\hline Cumulative Illness Rating Scale score & $5.7 \pm 3.8$ & $5.1 \pm 3.7$ \\
\hline Education (years) & $13.2 \pm 3.7^{* * * *}$ & $15.3 \pm 3.4$ \\
\hline Number of rooms in home & $4.9 \pm 1.7$ & $5.8 \pm 2.4$ \\
\hline White race & $21(77.8 \%)$ & $212(86.2 \%)$ \\
\hline Occupational category & & $28(11.4 \%)$ \\
\hline Higher executive & $1(3.7 \%)$ & $90(36.7 \%)$ \\
\hline Business manager & $10(37.0 \%)$ & $42(17.1 \%)$ \\
\hline Administrative & $4(14.8 \%)$ & $62(25.3 \%)$ \\
\hline Clerical/sales & $8(29.6 \%)$ & $13(5.3 \%)$ \\
\hline Skilled manual & $1(3.7 \%)$ & $5(2.0 \%)$ \\
\hline Machine operator & $2(7.4 \%)$ & $5(2.0 \%)$ \\
\hline Unskilled & $1(3.7 \%)$ & \\
\hline
\end{tabular}

Values are means $\pm \mathrm{SD}$.

$*$

$\mathrm{p}<0.01$

$* *$

$\mathrm{p}<0.05$

****

$p<0.01$

$\mathrm{p}<0.001$ (Chi-square or Student's t tests). 
Table 2

Tremor severity in smokers vs. non-smokers

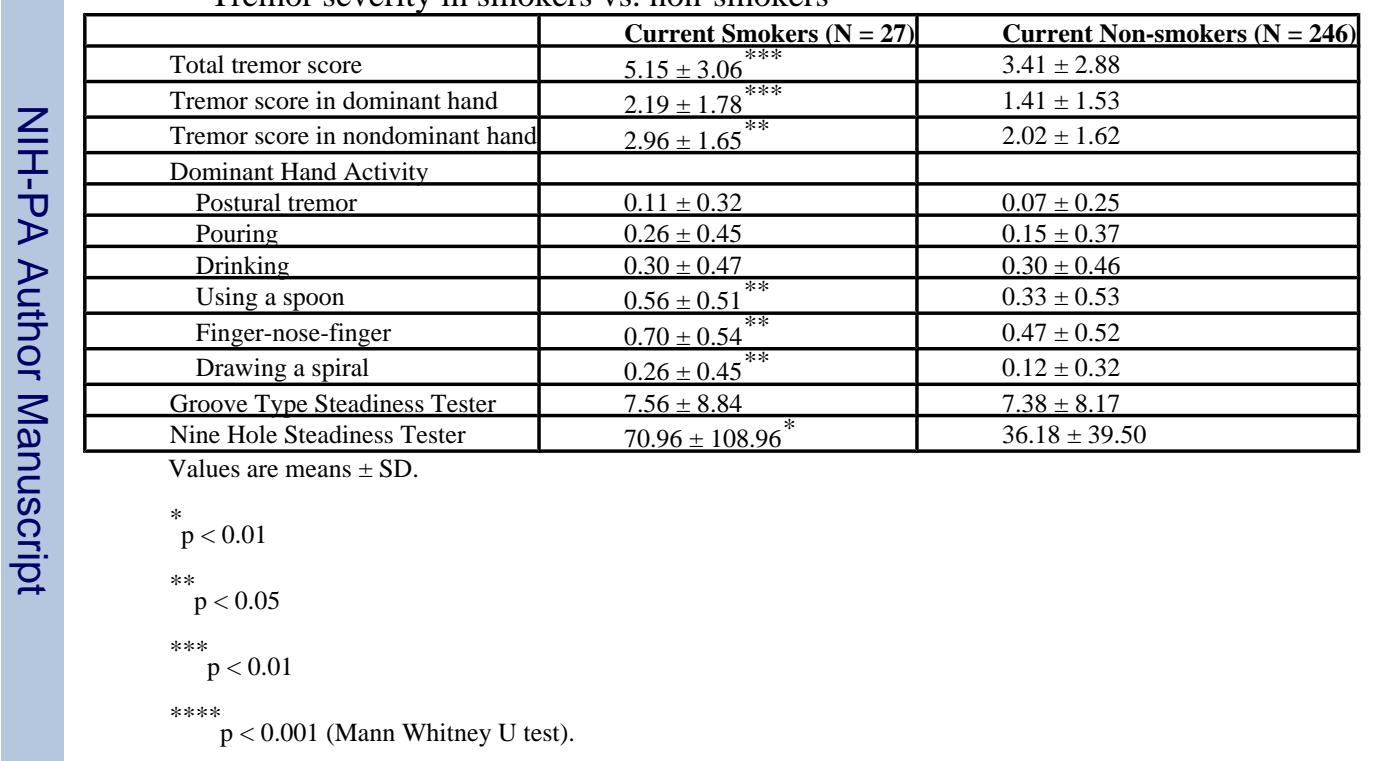


Table 3

Tremor severity in smokers vs. non-smokers stratified by gender

\begin{tabular}{|c|c|c|c|c|}
\hline & \multicolumn{2}{|l|}{ Men } & \multicolumn{2}{|l|}{ Women } \\
\hline & $\begin{array}{l}\text { Current Smokers } \\
\mathrm{N}=10\end{array}$ & $\begin{array}{l}\begin{array}{l}\text { Current Non- } \\
\text { smokers N }=94\end{array} \\
\end{array}$ & $\begin{array}{l}\text { Current Smokers N = } \\
17\end{array}$ & $\begin{array}{l}\text { Current Non- } \\
\text { smokers N = 151 }\end{array}$ \\
\hline Total tremor score & $5.40 \pm 2.88$ & $4.39 \pm 3.39$ & $5.00 \pm 3.24^{* * *}$ & $2.81 \pm 2.34$ \\
\hline Tremor score in dominant hand & $2.20 \pm 1.93$ & $1.98 \pm 1.73$ & $2.18 \pm 1.74^{* * *}$ & $1.06 \pm 1.28$ \\
\hline Tremor score in nondominant hand & $3.20 \pm 1.40^{*}$ & $2.41 \pm 1.87$ & $2.82 \pm 1.81^{\text {** }}$ & $1.77 \pm 1.40$ \\
\hline Dominant Hand Activity & & & & \\
\hline Postural tremor & $0.10 \pm 0.32$ & $0.11 \pm 0.31$ & $0.12 \pm 0.33$ & $0.04 \pm 0.20$ \\
\hline Pouring & $0.30 \pm 0.48$ & $0.20 \pm 0.40$ & $0.24 \pm 0.44$ & $0.11 \pm 0.34$ \\
\hline Drinking & $0.20 \pm 0.42$ & $0.39 \pm 0.49$ & $0.35 \pm 0.49$ & $0.23 \pm 0.42$ \\
\hline Using a spoon & $0.60 \pm 0.52$ & $0.46 \pm 0.54$ & $0.53 \pm 0.51^{* * *}$ & $0.25 \pm 0.51$ \\
\hline Finger-nose-finger & $0.60 \pm 0.52$ & $0.59 \pm 0.54$ & $0.76 \pm 0.56^{\text {*** }}$ & $0.40 \pm 0.49$ \\
\hline Drawing a spiral & $0.40 \pm 0.52$ & $0.23 \pm 0.42$ & $0.18 \pm 0.39^{* *}$ & $0.05 \pm 0.23$ \\
\hline Groove Type Steadiness Tester & $3.80 \pm 3.19$ & $8.00 \pm 9.18$ & $9.76 \pm 10.36$ & $6.92 \pm 7.45$ \\
\hline Nine Hole Steadiness Tester & $44.30 \pm 58.17$ & $38.80 \pm 37.45$ & $87.63 \pm 130.33^{* * *}$ & $33.64 \pm 39.11$ \\
\hline \multicolumn{5}{|l|}{ Values are means \pm SD. } \\
\hline \multicolumn{5}{|l|}{$* x<0.10$} \\
\hline \multicolumn{5}{|l|}{$* *$} \\
\hline \multicolumn{5}{|l|}{$* * * \quad p<0.01$} \\
\hline${ }^{* * * *} p<0.001$ (Mann Whitney & & & & \\
\hline
\end{tabular}


Linear regression analyses

Table 4

Dependent variable(s) in the linear regression model

beta and p value for the smoking variable only

Smoking (unadjusted model)

beta $=0.25, \mathrm{p}=0.002$

Smoking adjusted for age, gender, caffeine consumption per day in mg, taking a tremor-inducing

beta $=0.21, \mathrm{p}=0.01$

education. number of rooms in home, white race, occupational category

Smoking adjusted for blood lead concentration

beta $=0.24, p=0.009$

Smoking adjusted for blood harmane concentration

Smoking adjusted for serum $\mathrm{p}, \mathrm{p}^{\prime}$-DDE concentration

beta $=0.22, \mathrm{p}=0.019$

Smoking adjusted for serum $p, p^{\prime}$-DDT concentration

Smoking adjusted for serum $\beta$-hexachlorocyclohexane concentration

beta $=0.20, p=0.03$

Smoking adjusted for serum oxychlordane concentration

beta $=0.20, \mathrm{p}=0.03$

Smoking adjusted for serum trans-nonachlor concentration

beta $=0.21, \mathrm{p}=0.03$

\begin{tabular}{l|l} 
ation & beta $=0.20, \mathrm{p}=0.03$ \\
\hline
\end{tabular}

Smoking adjusted for serum dieldrin concentration

All beta values are for the smoking variable only and not for the entire model.

beta $=0.22, p=0.02$

